\title{
openheart Impact of emergency medical service delays on time to reperfusion and mortality in STEMI
}

Ahmad Alrawashdeh (D) , ${ }^{1,2}$ Ziad Nehme, ${ }^{1,3,4}$ Brett Williams, ${ }^{1}$ Karen Smith, ${ }^{1,3,4}$ Angela Brennan, ${ }^{3,5}$ Diem T Dinh, ${ }^{3}$ Danny Liew, ${ }^{3}$ Jeffrey Lefkovits, ${ }^{3,6}$ Dion Stub (1) $3,5,6,7$

To cite: Alrawashdeh $A$, Nehme Z, Williams B, et al. Impact of emergency medical service delays on time to reperfusion and mortality in STEMI. Open Heart 2021;8:e001654. doi:10.1136/ openhrt-2021-001654

Received 28 March 2021 Revised 14 April 2021 Accepted 16 April 2021

Check for updates

C) Author(s) (or their employer(s)) 2021. Re-use permitted under CC BY-NC. No commercial re-use. See rights and permissions. Published by BMJ.

'Department of Paramedicine, Monash University, Frankston, Victoria, Australia

${ }^{2}$ Department of Allied Medical Sciences, Jordan University of Science and Technology, Irbid, Jordan

${ }^{3}$ School of Public Health and Preventive Medicine, Monash University, Melbourne, Victoria, Australia

${ }^{4}$ Ambulance Victoria, Blackburn North, Victoria, Australia

${ }^{5}$ Alfred Hospital, Prahran, Victoria, Australia

${ }^{6}$ Department of Cardiology, Royal Melbourne Hospital, Melbourne, Victoria, Australia ${ }^{7}$ Baker IDI Heart and Diabetes Institute, Melbourne, Victoria, Australia

Correspondence to

A/Prof Dion Stub; d.stub@alfred. org.au

\section{ABSTRACT}

Objectives To explore the relationship between emergency medical service (EMS) delay time, overall time to reperfusion and clinical outcome in patients with ST-elevation myocardial infarction (STEMI) undergoing primary percutaneous coronary intervention (PPCI). Methods This was a retrospective observational study of 2976 patients with STEMI who presented to EMS and underwent PPCI between January 2014 and December 2017. We performed multivariable logistic models to assess the relationship between EMS delay time and 30-day mortality and to identify factors associated with system delay time.

Results EMS delay time accounted for the first half of total system delay (median $=59 \mathrm{~min}(\mathrm{IQR}=48-77))$. Compared with those who survived, those who died had longer median EMS delay times (59 (IQR=11-74) vs $74(\mathrm{IQR}=57-98), \mathrm{p}<0.001)$. EMS delay time was independently associated with a higher risk of mortality (adjusted OR 1.20; 95\% Cl 1.02 to 1.40 , for every 30 min increase), largely driven by complicated patients with cardiogenic shock or cardiac arrest. Independent predictors of longer EMS delay times were older age, women, cardiogenic shock, cardiac arrest, prehospital notification and intensive care management. Although longer EMS delay times were associated with shorter door-to-balloon times, total system delay times increased with increasing EMS delay times.

Conclusion Increasing EMS delay times, particularly those result from haemodynamic complications, increase total time to reperfusion and are associated with 30 day mortality after STEMI. All efforts should be made to monitor and reduce EMS delay times for timely reperfusion and better outcome.

\section{INTRODUCTION}

There is a well-defined relationship between time to treatment and clinical outcomes in acute ST-elevation myocardial infarction (STEMI) undergoing primary percutaneous coronary intervention (PPCI).$^{1-3}$ Reducing total system delays between first medical contact and reperfusion has been associated with better patient outcomes and is recommended by international guidelines. ${ }^{4-7}$

\section{Key messages}

What is already known about this subject?

- Emergency medical service (EMS) diagnosis and prehospital notification of ST-elevation myocardial infarction (STEMI) have been repeatedly shown to reduce door-to-balloon time (DTB) time and mortality. However, little attention has been given to understanding how emergency medical EMS delays influence time to treatment and patient outcome after STEMI.

What does this study add?

- The results of this statewide observational study demonstrate that longer EMS delay times are independently associated with higher mortality. However, this relationship is mainly driven by complicated patients with cardiogenic shock and out-of-hospital cardiac arrest.

- Although longer EMS delays driven by the presence of intensive care paramedics and transmitting a 12lead ECG were associated with shorter DTB times, increasing EMS delays were also associated with longer overall system delay times.

How might this impact on clinical practice?

- Our findings suggest a crucial need for performance improvement interventions aiming at reducing and optimising EMS delay, particularly among complicated patients with cardiogenic shock and out-ofhospital cardiac arrest.

Traditionally, however, significant emphasis has been placed on improving door-toballoon (DTB) times, and comparatively less attention has been given to understanding how emergency medical service (EMS) delays influence time to treatment and patient outcome after STEMI.

EMS diagnosis and prehospital notification of STEMI have been repeatedly shown to reduce DTB time. ${ }^{89}$ As such there has been increasing emphasis on the role of EMS in STEMI systems of care. Patients with STEMI using EMS have noticeably increased to more than $60 \%$ in developed countries. ${ }^{1011}$ 
However, only about $50 \%$ of EMS users receive reperfusion within the recommended system delay time to treatment. Furthermore, prehospital delay times have not improved over recent decades. ${ }^{11} 12$ In fact, a recent systematic review has shown a four-fold global variation in EMS system delays for STEMI patients. ${ }^{12}$ Despite this, the impact of EMS delay times on time to treatment and patient outcomes have not been well established in patients with STEMI.

In this observational study, we sought to (1) assess the relationship between EMS delay times and 30-day mortality rate after PPCI, (2) identify factors associated with EMS delay times and (3) assess the relationship between EMS delay times, DTB time and total system delay time.

\section{METHODS}

\section{Design and setting}

We performed a multicentre retrospective observational study of consecutive patients with STEMI who presented to EMS and underwent PPCI in Victoria, Australia, between January 2014 and December 2017. This study was approved by the Monash University Human Research Ethics Committee (project number: 9557).

The STEMI system of care in Victoria comprises 13 public and 17 private PCI-capable hospitals and one EMS agency, Ambulance Victoria. Ninety per cent of patients with STEMI are taken to a public hospital for PPCI. ${ }^{13}$ The EMS operates a two-tiered system involving advanced life support and mobile intensive care ambulance (MICA) paramedics. The clinical practice guidelines of Ambulance Victoria recommend MICA paramedics manage and transport suspected patients with STEMI directly to a PPCI-capable hospital within $60 \mathrm{~min}$ of diagnosis. Hospital prenotification and transmission of the 12-lead ECG activates the cardiac catheterisation laboratory. ${ }^{14}$ All MICA were equipped with 12-lead ECG capable cardiac monitors and wireless transmission technology in 2009. This technology then became available to all ambulances in 2016. Treatment guidelines authorise the use of aspirin, nitrates and opioids by all paramedics. Intensive care paramedics are also authorised to administer a heparin bolus prior to PPCI. Where appropriate, patients are directly triaged to the cardiac catheterisation laboratory bypassing the emergency department at the interventional hospital.

\section{Data source and participants}

The Victorian Cardiac Outcomes Registry is a clinical quality registry that prospectively captures demographic and clinical data for all PCI procedures in Victorian hospitals. The registry has been described in detail previously. For this study, we included patients undergoing PPCI after acute onset of STEMI (symptom onset within 12 hours) following presentation to EMS over the study period. We excluded patients self-presenting to hospital and those with a secondary transfer after being triaged to a noninterventional hospital. We extracted patient demographics, comorbidity, clinical characteristics, 30-day mortality and date and time of symptom onset and PPCI reperfusion. Thirty-day mortality was collected during telephone follow-up 30 days post-PPCI by the participating hospital. Registry data also undergo linkage with prehospital care records captured electronically by Ambulance Victoria. The prehospital data include EMS time intervals and EMS management (ie, use of prehospital 12-lead ECG and presence of MICA on scene).

\section{Definitions and outcome measures}

The primary outcome measure was 30-day mortality, and the principal independent variable was EMS delay time, defined as the total duration between the call to EMS and arrival at hospital. The secondary outcome measure was total system delay time consisting of both EMS delay time and DTB time. DTB time was defined as the time between arrival at hospital and balloon inflation. We defined patient delay time as the time between symptom onset and call to EMS. We also further stratified the total system delay into subcomponents, including EMS response time, scene time and transport time, door-to-cath lab time and cath lab-to-balloon time (figure 1).

\section{Patient and public involvement}

Patients and the public were not involved in the study design, analysis, interpretation and writing of the study.

\section{Statistical analysis}

For the descriptive statistics, we stratified EMS delay time at its median ( $\leq 60 \mathrm{~min}$ or $>60 \mathrm{~min}$ ) and compared baseline and clinical characteristics across groups. We also compared baseline characteristics across patients who survived and did not survive to 30 days post-PPCI. Categorical data were expressed as frequencies and percentages and were compared between groups using the $\chi^{2}$ test. Continuous variables were presented as mean and SD or median and IQR as appropriate for their distribution and were compared across groups using the Student's t-test or Kruskal-Wallis test, respectively.

To investigate the impact of EMS delay time on 30-day mortality, we fitted multivariable logistic regression models. In the analysis, we included age (per 10-year increase), female sex, comorbidity (previous revascularisation with PCI or coronary artery bypass grafting (diabetes, peripheral vascular disease, cerebrovascular disease and chronic use of oral anticoagulant therapy), clinical characteristics prior to PCI (cardiogenic shock, out of hospital cardiac arrest (OHCA) and anterior infarction), status of the prehospital 12-lead ECG (not taken, taken and not transmitted and taken and transmitted), presence of MICA paramedics on scene, and the delay time intervals to reperfusion. We entered patient delay time, EMS delay time and DTB time as continuous variables per $30 \mathrm{~min}$ increase. We verified the linearity between delay time intervals and mortality using multivariable fractional polynomial models. In a sensitivity 


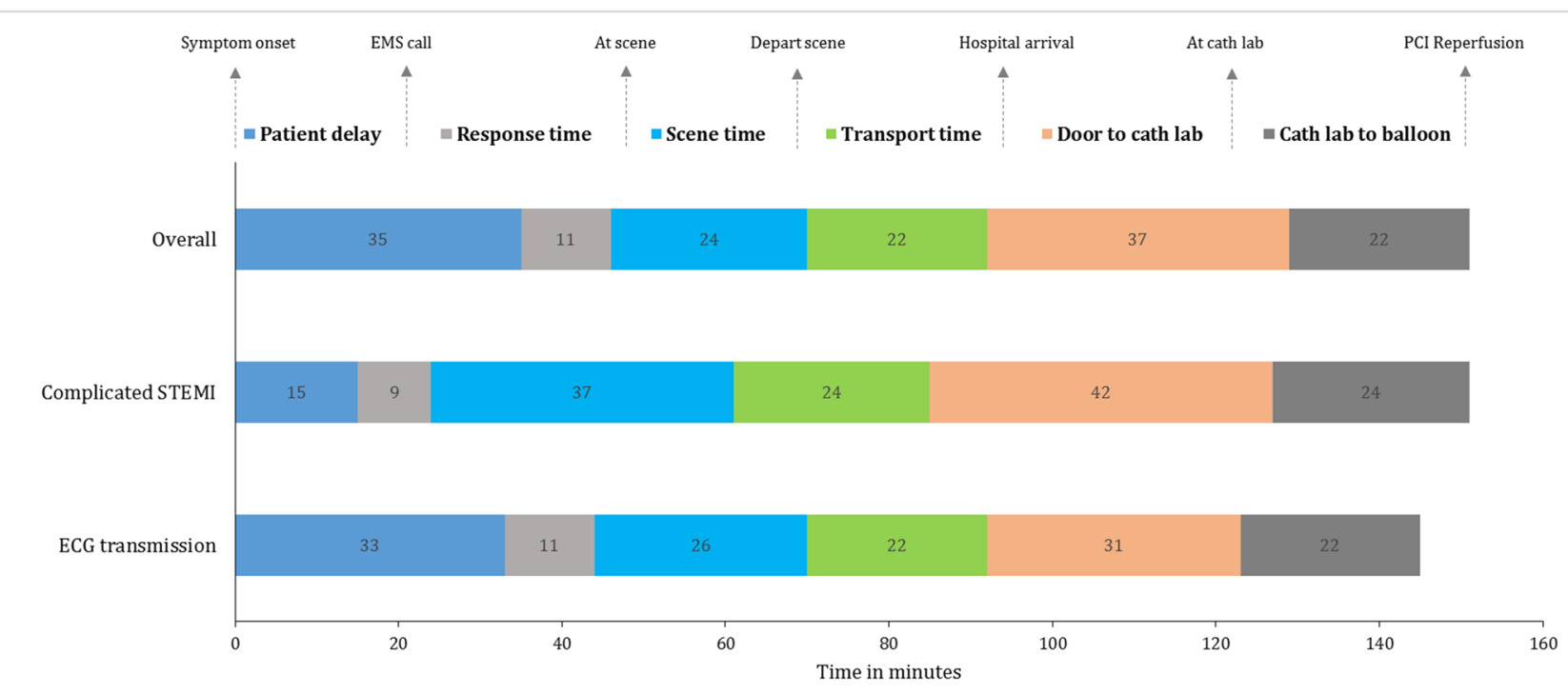

Figure 1 Duration of subcomponents of time to treatment. EMS, emergency medical service; STEMI, ST-elevation myocardial infarction.

analysis, we repeated the model above after excluding patients with cardiogenic shock and OHCA, as these patients typically have higher mortality rates and longer system delays. As some evidence indicates that the relationship between system delays and mortality in STEMI is augmented by shorter patient delay times, ${ }^{35}$ we also tested an interaction term between patient delay time ( $\leq 30$ or $>30 \mathrm{~min}$ ) and EMS delay time.

We conducted multivariable logistic regression models to identify factors associated with EMS delays and DTB time. We stratified EMS delay time and DTB time according to their medians $(\leq 60 \mathrm{~min}$ or $>60 \mathrm{~min}$ ). The variables included in the models were age (per 10-year increase), female sex, comorbidity, clinical characteristics, status of prehospital 12-lead ECG, MICA on scene, time and date of EMS call and the appropriate delay time intervals.

We also performed multivariable logistic regression models to assess the relationship between EMS subcomponents on receiving a delayed DTB time $(>60 \mathrm{~min})$ and total system delay time ( $>120 \mathrm{~min})$, while adjusting for age and gender. We dichotomised EMS subcomponents according to the pooled weighted means of a recent meta-analysis, including response time $(>11 \mathrm{~min})$, scene time $(>20 \mathrm{~min})$ and transport time $(>21 \mathrm{~min}) .{ }^{12}$ The results of all logistic regression models were presented as OR and 95\% CIs. All analyses were conducted using Stata Statistical Software, V.15.1 (StataCorp, 2017, College Station, Texas, USA). All $\mathrm{p}$ values were two-tailed, and a $p$ value $<0.05$ was considered significant in all analyses.

\section{RESULTS}

\section{Population}

Over the study period, a total of 2976 patients with STEMI presented to Ambulance Victoria and underwent PPCI at an interventional hospital. The mean age was
$62.5(\mathrm{SD}=12.5)$ and $19.7 \%$ were women. Five hundred and fifty $(18.5 \%)$ patients had haemodynamic complications with either cardiogenic shock $(\mathrm{n}=352 ; 11.8 \%)$ and/ or OHCA ( $\mathrm{n}=383,12.9 \%)$. A prehospital 12-lead ECG was taken in $74.9 \%$ and transmitted in $48.9 \%$. Table 1 shows the difference in system delay time and mortality in our population.

Figure 1 shows the average of subcomponents of total time to reperfusion by different groups. For total population, median patient delay time was $35 \mathrm{~min}(\mathrm{IQR}=10$ 96), median EMS delay time was $59 \mathrm{~min}$ ( $\mathrm{IQR}=48-77$ ), median DTB time was $61 \mathrm{~min}(\mathrm{IQR}=43-88)$, and median total system delay time was $127 \mathrm{~min}(\mathrm{IQR}=104-159)$. The proportion of patients who received a system delay time of $\leq 90 \mathrm{~min}$ and $\leq 120 \mathrm{~min}$ was $11.7 \%$ and $43.4 \%$, respectively. DTB was significantly improved with prehospital notification, median DTB $51 \mathrm{~min}$ (IQR 37-70) versus 75 mins (IQR 52-105), $\mathrm{p}<0.01$.

Among the overall population, 234 (7.9\%) patients died within 30 days of PPCI. Compared with those who survived, those who died had longer median EMS delay times (59 min (IQR=11-74) vs $74 \mathrm{~min} \quad(\mathrm{IQR}=57$ $98), \mathrm{p}<0.001)$ and DTB times $(60 \mathrm{~min}(\mathrm{IQR}=42-86)$ vs 78 min $([\mathrm{IQR}=54-110), \mathrm{p}<0.001)$ and shorter median patient delay times $(37 \mathrm{~min} \quad(\mathrm{IQR}=13-100)$ vs $13 \mathrm{~min}$ $(\mathrm{IQR}=4-55)$, $\mathrm{p}<0.001)$. Mortality was $31.5 \%$ among patients with haemodynamic complications compared with $2.5 \%$ among those patients without haemodynamic complications.

\section{Impact of EMS delay time on 30-day mortality}

In the multivariable analysis (table 2), EMS delay time was independently associated with 30-day mortality in the overall population, with an adjusted OR (aOR) of 1.20 (95\% CI 1.02 to $1.40, \mathrm{p}=0.025$ ) for every $30 \mathrm{~min}$ increase. Figure 2 shows the adjusted predicted mortality by EMS 
Table 1 Baseline characteristics and differences in EMS delay times and 30-day mortality

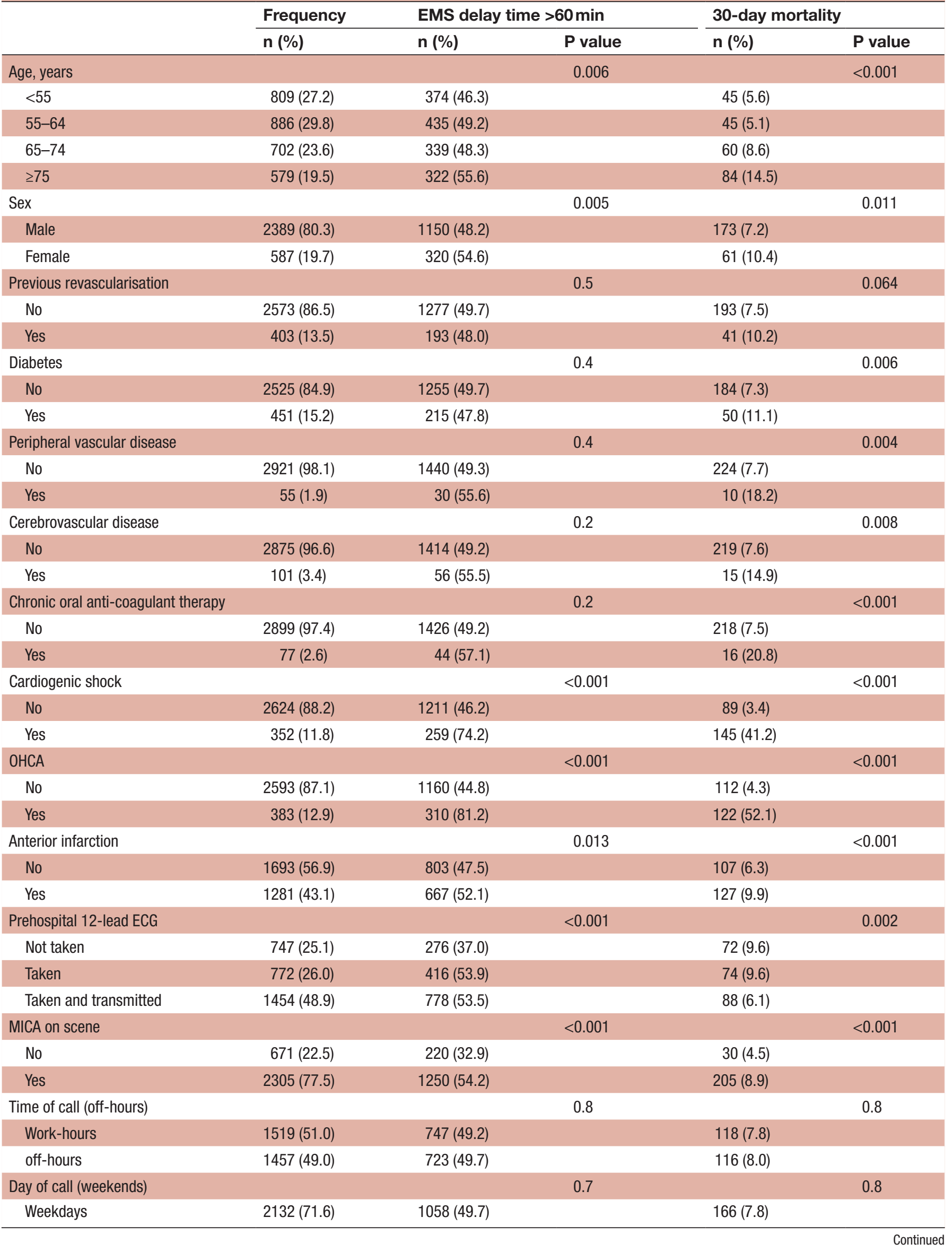


Table 1 Continued

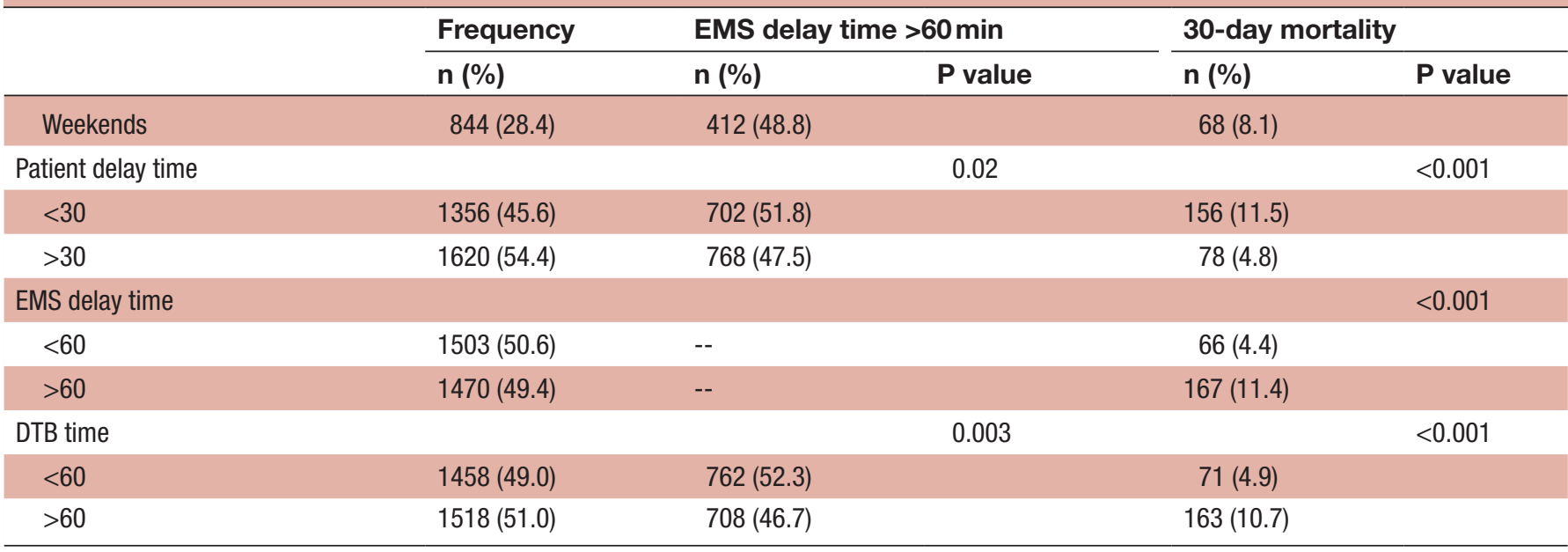

DTB, door-to-balloon; EMS, emergency medical service; MICA, mobile intensive care ambulance; OHCA, out-of-hospital cardiac arrest.

Table 2 Adjusted association between study variables and 30-day mortality rate

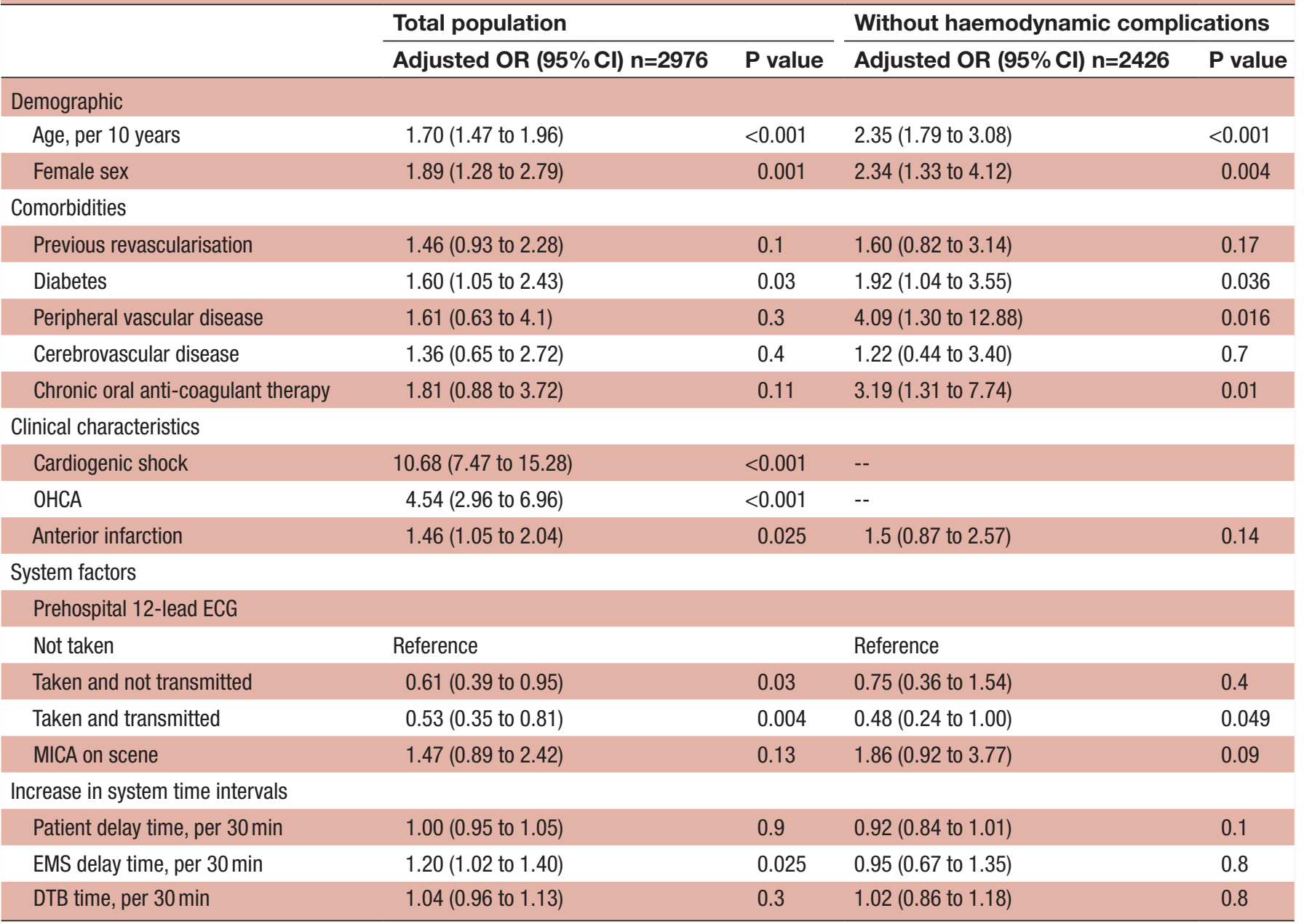

The interaction term between patient delay $>30 \mathrm{~min}$ and EMS delay time was significant in the total population (adjusted OR $0.81 ; 95 \% \mathrm{Cl}$ 0.70 to $0.92 ; p=0.001$ ).

DTB, door-to-balloon; EMS, emergency medical service; MICA, mobile intensive care ambulance; OHCA, out-of-hospital cardiac arrest. 


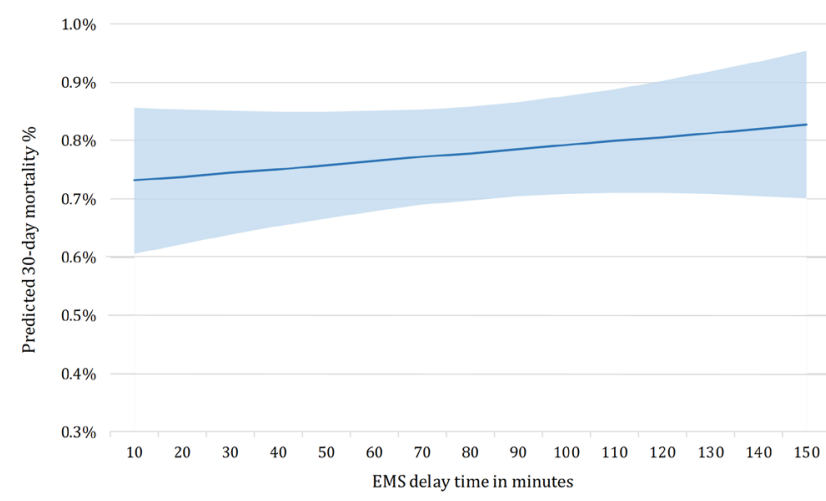

Figure 2 Adjusted predicted mortality rate with $95 \% \mathrm{Cl}$ (shaded area) associated with EMS delay time. EMS,emergency medical service.

delay time. However, longer EMS delay times were not significantly associated with mortality among patients without cardiogenic shock or OHCA (aOR $0.95 ; 95 \%$ CI 0.67 to $1.35 ; \mathrm{p}=0.8$ ).

\section{Factors associated with EMS delay time}

The aOR of receiving an EMS delay time $>60$ min were higher among patients with older age (per 10-year increase, aOR 1.11; 95\% CI 1.04 to 1.18), female sex (aOR 1.43; $95 \%$ CI 1.17 to 1.74 ), cardiogenic shock (aOR $1.91 ; 95 \%$ CI 1.44 to 2.54 ) and OHCA (aOR 4.21; $95 \%$ CI 3.13 to 5.67). A longer EMS delay time was also associated with transmitting a prehospital 12-lead ECG and presence of MICA on scene (table 3). Comorbidity, time and day of call and patient delay time were not associated with EMS delay time.

Impact of EMS delay time on DTB time and total system delay time Factors associated with longer DTB times are presented in table 3 . The aOR of receiving a DTB time $>60$ min were lower among patients who had a transmitted prehospital 12-lead ECG (aOR 0.39; 95\% CI 0.31 to 0.49 ) and MICA on scene (aOR $0.45 ; 95 \%$ CI 0.36 to 0.56 ). Increasing EMS delay times were inversely associated with DTB times. An EMS delay time $>60 \mathrm{~min}$ reduced the odds of experiencing a DTB time $>60$ min $(\mathrm{aOR} 0.80 ; 95 \% \mathrm{CI}$ 0.68 to 0.95$)$.

Table 3 Multivariable logistic regression of factors associated with system time intervals

\begin{tabular}{|c|c|c|c|c|}
\hline & \multicolumn{2}{|c|}{ EMS delay time $>60 \mathrm{~min}$} & \multicolumn{2}{|c|}{ DTB time $>60 \mathrm{~min}$} \\
\hline & aOR $(95 \% \mathrm{Cl})$ & $P$ value & aOR (95\% Cl) & P value \\
\hline \multicolumn{5}{|l|}{ Demographic } \\
\hline Female sex & $1.43(1.17$ to 1.74$)$ & $<0.001$ & 1.06 (0.86 to 1.31$)$ & 0.6 \\
\hline \multicolumn{5}{|l|}{ Comorbidities } \\
\hline Diabetes & 0.94 (0.76 to 1.17$)$ & 0.6 & 1.30 (1.04 to 1.63$)$ & 0.022 \\
\hline Peripheral vascular disease & 1.31 (0.73 to 2.35$)$ & 0.4 & $0.53(0.29$ to 0.97$)$ & 0.039 \\
\hline Cerebrovascular disease & 1.25 (0.82 to 1.93$)$ & 0.3 & $1.17(0.74$ to 1.85$)$ & 0.5 \\
\hline Chronic oral anti-coagulant therapy & 1.08 (0.66 to 1.77$)$ & 0.8 & 1.54 (0.92 to 2.58) & 0.1 \\
\hline Anterior infarction & $1.12(0.96$ to 1.31$)$ & 0.2 & 1.36 (1.15 to 1.6$)$ & $<0.001$ \\
\hline \multicolumn{5}{|l|}{ System factors } \\
\hline \multicolumn{5}{|l|}{ Prehospital 12-lead ECG } \\
\hline Not taken & Reference & & Reference & \\
\hline Taken and not transmitted & 1.67 (1.33 to 2.10$)$ & $<0.001$ & 1.08 (0.85 to 1.36$)$ & 0.5 \\
\hline Taken and transmitted & 1.68 (1.36 to 2.08$)$ & $<0.001$ & 0.39 (0.31 to 0.49$)$ & $<0.001$ \\
\hline MICA on scene & 1.68 (1.36 to 2.07$)$ & $<0.001$ & 0.45 (0.36 to 0.56$)$ & $<0.001$ \\
\hline Time of call (off-hours) & 1.06 (0.91 to 1.24$)$ & 0.5 & 2.56 (2.18 to 3.01$)$ & $<0.001$ \\
\hline Day of call (weekends) & 0.95 (0.80 to 1.12) & 0.5 & 1.97 (1.65 to 2.36) & $<0.001$ \\
\hline
\end{tabular}

aOR, adjusted OR; DTB, door-to-balloon; EMS, emergency medical service; MICA, mobile intensive care ambulance; OHCA, out-of-hospital cardiac arrest. 
Table 4 Relationship between EMS time intervals, DTB time and total system delay time

\begin{tabular}{|c|c|c|c|c|}
\hline & DTB time $>60 \mathrm{~m}$ & & System delay time & $\min$ \\
\hline & aOR $(95 \% \mathrm{Cl})$ & $P$ value & aOR $(95 \% \mathrm{Cl})$ & $P$ value \\
\hline With haemodynamic compli & & & & \\
\hline Response time $>11 \mathrm{~min}$ & 0.78 (0.53 to 1.16$)$ & 0.22 & 1.74 (0.94 to 3.22$)$ & 0.076 \\
\hline Scene time $>20 \mathrm{~min}$ & $1.36(0.84$ to 2.20$)$ & 0.211 & $8.30(4.15$ to 16.60$)$ & $<0.001$ \\
\hline Transport time $>21 \mathrm{~min}$ & 0.71 (0.50 to 1.02$)$ & 0.064 & 7.26 (4 to 13.14$)$ & $<0.001$ \\
\hline DTB $>60 \mathrm{~min}$ & -- & & 45.31 (22.56 to 91.01) & $<0.001$ \\
\hline Without haemodynamic con & & & & \\
\hline Response time $>11 \mathrm{~min}$ & $1.00(0.84$ to 1.19$)$ & 0.991 & 3.54 (2.74 to 4.57$)$ & $<0.001$ \\
\hline Scene time $>20 \mathrm{~min}$ & 0.81 (0.69 to 0.96$)$ & 0.016 & 3.29 (2.57 to 4.20$)$ & $<0.001$ \\
\hline Transport time $>21 \mathrm{~min}$ & 0.66 (0.56 to 0.78$)$ & $<0.001$ & 10.63 (7.76 to 14.55$)$ & $<0.001$ \\
\hline DTB $>60$ min & -- & & 68.06 (48.66 to 95.21) & $<0.001$ \\
\hline
\end{tabular}

Adjusted for age and sex.

aOR, adjusted OR; DTB, door-to-balloon; EMS, emergency medical service.

The relationship between EMS time intervals and DTB time and total system delay time is shown in table 4 . In patients with haemodynamic compromise, scene times $>20$ min and transport time $>21$ min were significantly associated with total system delays $>120 \mathrm{~min}$. In patients without haemodynamic compromise, scene times $>20$ min and transport time $>21 \mathrm{~min}$ were inversely associated with DTB times $>60 \mathrm{~min}$. However, increasing EMS delay intervals and DTB was also associated with increased total system delays $>120 \mathrm{~min}$.

\section{DISCUSSION}

This state-wide observational study explored the impact of EMS time delays on patient outcome and total time to PPCI after STEMI. Our results demonstrate that longer EMS delay times are independently associated with higher mortality. This relationship is mainly driven by complicated patients with cardiogenic shock and OHCA, who have significantly higher mortality and longer EMS delay times. Although longer EMS delay times, particularly those resulting from prehospital notification and intensive care management, were partly offset by shorter DTB times, total system delays to reperfusion increased with increasing EMS delay times.

In our recent meta-analysis of diverse studies including directly transported patients to an interventional hospital, we failed to show a significant relationship between EMS delay times and short-term mortality. ${ }^{12}$ The results of the meta-analysis were obtained from selected STEMI populations with a low pooled mortality rate of $4.1 \%$. However, our findings are supported by a Danish multicentre study of unselected cohort of 6209 patients, involving both EMS directly referred and interhospital transferred STEMI, which found that prehospital system delay time was independently associated with mortality. ${ }^{5}$ Similarly, a UK study of STEMI patients admitted directly to interventional hospitals showed that EMS delays $>60$ min were associated with a higher risk of 30-day mortality. ${ }^{15}$ Yet, some of those patients had self-presented to the interventional hospitals.

In accordance with our findings, two recent large observational studies demonstrated a detrimental effect of reperfusion delays on outcome in complicated STEMI with cardiogenic shock and/or cardiac arrest. ${ }^{16}{ }^{17}$ In patient with uncomplicated STEMI, however, the impact of reperfusion delays on clinical outcome is less clear. ${ }^{18}$ This justifies the increasing emphasis on early reperfusion in patients with cardiogenic shock according to current recommendations from the European and American guidelines. ${ }^{6} 719$ Unfortunately, reperfusion delays in patients with haemodynamic instability have not improved despite a number of improvement strategies. For example, despite improvements in achieving targeted timely reperfusion between first medical contact and reperfusion ( $<90 \mathrm{~min}$ ) throughout the AHA Mission: Lifeline STEMI Systems Accelerator project, data from this project failed to show an improvement in achieving timely reperfusion for patients with cardiogenic shock who presented directly to interventional hospitals by EMS. ${ }^{16}$

Our analyses demonstrate that patients with cardiogenic shock experience additional delays in both EMS and hospital intervals. Reducing these delays is a challenge for EMS and hospital providers, ${ }^{20}$ who are required to stabilise patients before providing further treatments directed towards reperfusion. ${ }^{19}$ EMS providers may first need to perform additional interventions on-scene (ie, intravenous fluid, securing airways and cardiopulmonary resuscitation) to facilitate safe transportation. Some patients, however, may not quickly and effectively respond to prehospital interventions, which may hinder early STEMI diagnosis and hospital notification. Although managing haemodynamically unstable patients may create unavoidable delays, new strategies that streamline STEMI systems of care among critically ill patients may help minimise total system time to reperfusion. 
'Consistent with previous research, gender was associated with a small but significant EMS delay. ${ }^{21}$ Women have previously been described as having higher rates of incorrect triage and delay to first ECG. ${ }^{22}$ Ongoing education for EMS and medical teams are needed to address the gender gap in STEMI care'.

The present study confirms previous findings and contributes additional evidence that suggests the need for optimising EMS performance to guarantee timely reperfusion. ${ }^{2324}$ Although longer EMS delay times were associated with shorter DTB times, increasing EMS delays were also associated with longer overall system delay times. The advantage of using EMS in reducing DTB time may not be observed without advanced hospital notification of an incoming patient with STEMI. ${ }^{25}$ However, a metaanalysis found that the reduction in DTB time following an advanced hospital notification by EMS is widely varied between systems. ${ }^{9}$ This variation can be attributed to differences in hospital performance and strategies to confirm STEMI diagnosis and activate the cardiac catheterisation laboratory (ie, field activation or emergency department activation). ${ }^{26} 27$ Data from the Accelerator project suggest that activating cardiac catheterisation laboratory within $20 \mathrm{~min}$ of first medical contact is associated with shorter DTB times and lower mortality. ${ }^{28}$ In the current study, we did not have data on the timing of prehospital notification and, therefore, we could not examine its impact on total system delays.

However, our EMS delay time was about 10 min longer than the global weighted mean of $50 \mathrm{~min}^{.{ }^{12}}$ This additional delay likely occurred during scene time that could be explained by the additional treatments provided by intensive care paramedics or the need for MICA back-up in many cases. As suggested by our previous meta-analysis, eliminating this $10 \mathrm{~min}$ delay may result in a $10 \%$ increase in the percentage of patients receiving timely system reperfusion. ${ }^{12}$ Consistent with previous studies, ${ }^{29}{ }^{30}$ we found that while DTB time $>60 \mathrm{~min}$ had the strongest association with delayed system time to reperfusion, delayed EMS subcomponents beyond the weighted global means were also predictive of delayed system time to reperfusion. This emphasises the need for ongoing monitoring and reporting of EMS time delays in the management of patient with STEMIs. Just as reporting of DTB times has prompted hospitals to monitor performance and reduce delays, reporting of EMS delay may yield similar improvements to EMS performance in minimising delays to reperfusion and improving patient outcomes.

\section{LIMITATIONS}

The study findings should be interpreted with the following limitations in mind. First, this was an observational study that is susceptible to unmeasured confounding and should not be used to infer a causal relationship between EMS delay times and mortality. Since it is inappropriate to assess the effect of EMS delays in randomised trials, larger observational studies may be required to validate our findings. Second, the data were obtained from a clinical quality registry and, therefore, susceptible to informational bias as treating hospitals may not report data for atypical events. Third, we did not control for risk differences in procedural factors or in quality of care between the participating hospitals. ${ }^{27}$ Finally, the characteristics of the regional STEMI system of care in this study may differ than many other regions that limit the generalisability of our findings.

\section{CONCLUSION}

EMS time delays constitute the first half of total system delay to reperfusion and are independently associated with 30-day mortality after STEMI, particularly among patients with haemodynamic complications. Although some EMS delays may be reasonable to enhance prehospital care, and improve in-hospital performance, ongoing monitoring on all aspects of patient ischaemic times are important in optimising STEMI systems of care.

Contributors Study was conceived and initially designed by AA, ZN and DS. Statistical analysis and interpretation were performed by AA and ZN. Conduct was supervised by ZN, BW and DS. The manuscript was drafted by AA and all authors made revisions for intellectual property.

Funding ZN is supported by a National Health and Medical Research Council (NHMRC) Early Career Fellowship (number 1146809). DS is supported by National Heart Foundation Fellowship and Viertel Foundation award.

Competing interests None declared.

Patient consent for publication Not required.

Provenance and peer review Not commissioned; externally peer reviewed.

Data availability statement Data are available upon reasonable request. Data were obtained retrospectively from a registry as deidentified participant data.

Open access This is an open access article distributed in accordance with the Creative Commons Attribution Non Commercial (CC BY-NC 4.0) license, which permits others to distribute, remix, adapt, build upon this work non-commercially, and license their derivative works on different terms, provided the original work is properly cited, appropriate credit is given, any changes made indicated, and the use is non-commercial. See: http://creativecommons.org/licenses/by-nc/4.0/.

ORCID iDs

Ahmad Alrawashdeh http://orcid.org/0000-0003-2458-8200

Dion Stub http://orcid.org/0000-0001-8686-2709

\section{REFERENCES}

1 De Luca G, Suryapranata H, Ottervanger JP, et al. Time delay to treatment and mortality in primary angioplasty for acute myocardial infarction: every minute of delay counts. Circulation 2004;109:1223-5.

2 Nallamothu B, Fox KAA, Kennelly BM, et al. Relationship of treatment delays and mortality in patients undergoing fibrinolysis and primary percutaneous coronary intervention. the global registry of acute coronary events. Heart 2007;93:1552-5.

3 Foo CY, Bonsu KO, Nallamothu BK, et al. Coronary intervention door-to-balloon time and outcomes in ST-elevation myocardial infarction: a meta-analysis. Heart 2018;104:1362-9.

4 Lambert L, Brown K, Segal E, et al. Association between timeliness of reperfusion therapy and clinical outcomes in ST-elevation myocardial infarction. JAMA 2010;303:2148-55.

5 Terkelsen CJ, Sørensen JT, Maeng M, et al. System delay and mortality among patients with STEMI treated with primary percutaneous coronary intervention. JAMA 2010;304:763-71.

6 Ibanez B, James S, Agewall S, et al. 2017 ESC guidelines for the management of acute myocardial infarction in patients presenting with ST-segment elevation: the task force for the management of acute myocardial infarction in patients presenting with ST-segment 
elevation of the European Society of cardiology (ESC). Eur Heart $J$ 2018;39:119-77.

7 O'Gara PT, Kushner FG, Ascheim DD, et al. 2013 ACCF/AHA guideline for the management of ST-elevation myocardial infarction: a report of the American College of cardiology Foundation/American heart association Task force on practice guidelines. Circulation 2013;127:e362-425

8 Quinn T, Johnsen S, Gale CP, et al. Effects of prehospital 12-lead ECG on processes of care and mortality in acute coronary syndrome: a linked cohort study from the myocardial ischaemia national audit project. Heart 2014;100:944-50.

9 Nam J, Caners K, Bowen JM, et al. Systematic review and metaanalysis of the benefits of out-of-hospital 12-lead ECG and advance notification in ST-segment elevation myocardial infarction patients. Ann Emerg Med 2014;64:176-86. 86.e1-9.

10 Mathews R, Peterson ED, Li S, et al. Use of emergency medical service transport among patients with ST-segment-elevation myocardial infarction: findings from the National cardiovascular data registry acute coronary treatment intervention outcomes network Registry-Get with the guidelines. Circulation 2011:124:154-63.

11 McGinn AP, Rosamond WD, Goff DC, et al. Trends in prehospital delay time and use of emergency medical services for acute myocardial infarction: experience in 4 us communities from 19872000. Am Heart J 2005; 150:392-400.

12 Alrawashdeh A, Nehme Z, Williams B, et al. Emergency medical service delays in ST-elevation myocardial infarction: a meta-analysis. Heart 2020;106:365-73.

13 Stub D, Lefkovits J, Brennan AL, et al. The establishment of the Victorian cardiac outcomes registry (VCOR): monitoring and optimising outcomes for cardiac patients in Victoria. Heart Lung Circ 2018;27:451-63.

14 Hutchison AW, Malaiapan Y, Jarvie I, et al. Prehospital 12-lead ECG to triage ST-elevation myocardial infarction and emergency department activation of the infarct team significantly improves door-to-balloon times: ambulance Victoria and MonashHEART acute myocardial infarction (MonAMI) 12-lead ECG project. Circ Cardiovasc Interv 2009;2:528-34.

15 Varcoe RW, Clayton TC, Gray HH, et al. Impact of call-toballoon time on 30 -day mortality in contemporary practice. Heart 2017; 103:117-24.

16 Kochar A, Al-Khalidi HR, Hansen SM, et al. Delays in primary percutaneous coronary intervention in ST-segment elevation myocardial infarction patients presenting with cardiogenic shock. JACC Cardiovasc Interv 2018;11:1824-33.

17 Scholz KH, Maier SKG, Maier LS, et al. Impact of treatment delay on mortality in ST-segment elevation myocardial infarction (STEMI) patients presenting with and without haemodynamic instability: results from the German prospective, multicentre FITT-STEMI trial. Eur Heart J 2018;39:1065-74.

18 Brodie BR, Stuckey TD, Muncy DB, et al. Importance of time-toreperfusion in patients with acute myocardial infarction with and without cardiogenic shock treated with primary percutaneous coronary intervention. Am Heart J 2003;145:708-15.

19 Thiele H, Ohman EM, de Waha-Thiele S, et al. Management of cardiogenic shock complicating myocardial infarction: an update 2019. Eur Heart J 2019;40:2671-83.

20 Noaman S, Andrianopoulos N, Brennan AL, et al. Outcomes of cardiogenic shock complicating acute coronary syndromes. Catheter Cardiovasc Interv 2020;96:E257-67.

21 Stehli J, Martin C, Brennan A, et al. Sex differences persist in time to presentation, revascularization, and mortality in myocardial infarction treated with percutaneous coronary intervention. J Am Heart Assoc 2019;8:e012161.

22 Benamer H, Bataille S, Tafflet M, et al. Longer pre-hospital delays and higher mortality in women with STEMI: the e-MUST registry. Eurolntervention 2016;12: $542-9$.

23 Studnek JR, Garvey L, Blackwell T, et al. Association between prehospital time intervals and ST-elevation myocardial infarction system performance. Circulation 2010;122:1464-9.

24 Cheskes S, Turner L, Foggett R, et al. Paramedic contact to balloon in less than 90 minutes: a successful strategy for ST-segment elevation myocardial infarction bypass to primary percutaneous coronary intervention in a Canadian emergency medical system. Prehosp Emerg Care 2011:15:490-8.

25 Afolabi BA, Novaro GM, Pinski SL, et al. Use of the prehospital ECG improves door-to-balloon times in ST segment elevation myocardial infarction irrespective of time of day or day of week. Emerg Med $J$ 2007;24:588-91.

26 Pedersen SH, Galatius S, Hansen PR, et al. Field triage reduces treatment delay and improves long-term clinical outcome in patients with acute ST-segment elevation myocardial infarction treated with primary percutaneous coronary intervention. J Am Coll Cardiol 2009;54:2296-302

27 Nallamothu BK, Wang Y, Magid DJ, et al. Relation between hospital specialization with primary percutaneous coronary intervention and clinical outcomes in ST-segment elevation myocardial infarction: National Registry of Myocardial Infarction-4 analysis. Circulation 2006;113:222-9.

28 Jollis JG, Al-Khalidi HR, Roettig ML, et al. Impact of regionalization of ST-segment-elevation myocardial infarction care on treatment times and outcomes for emergency medical services-transported patients presenting to hospitals with percutaneous coronary intervention: mission: lifeline accelerator-2. Circulation 2018;137:376-87.

29 Clark CL, Berman AD, McHugh A, et al. Hospital process intervals, not EMS time intervals, are the most important predictors of rapid reperfusion in EMS patients with ST-segment elevation myocardial infarction. Prehosp Emerg Care 2012;16:115-20.

30 Chughtai $\mathrm{H}$, Ratner D, Pozo M, et al. Prehospital delay and its impact on time to treatment in ST-elevation myocardial infarction. $A m ~ J$ Emerg Med 2011;29:396-400. 